\title{
Interpolação em 3D de Imagens de Tomografia Computadorizada usando Equações Diferenciais
}

S.R. PIRES1 1 Faculdades Alves Faria, Av. Perimetral Norte, 4129, Vila João Vaz, 74.445-190 Goiânia, GO, Brasil.

C.A.Z. BARCELOS ${ }^{2}$, M.A. BATISTA, E.L. FlÔRES, G.A. CARRIJO, A.C.P. VEIGA ${ }^{3}$, Universidade Federal de Uberlândia, Campus Santa Mônica, R. João Naves de Ávila, 2160, Cx.P. 593, 38.400-902 Uberlândia, MG, Brasil.

Resumo. A visualização em 3D do resultado de um exame de tomografia computadorizada (TC) aumenta a qualidade do diagnóstico médico e, conseqüentemente, a eficácia do tratamento. Para obter imagens com alta qualidade é necessário que as fatias (imagem dos resultados da TC) estejam muito próximas uma das outras, aumentando o tempo de exposição do paciente aos raios-X. Motivado pelo objetivo de obter um adequado balanceamento entre o tempo de exposição do paciente aos raios- $\mathrm{X}$ e a qualidade da visualização, este artigo apresenta o retoque digital como uma forma de interpolação em 3D para as fatias, permitindo a visualização em $3 \mathrm{D}$ das estruturas do corpo humano. O retoque é realizado pelo uso de equações diferenciais não lineares e este trabalho propõe um método de interpolação para preencher as regiões vazias entre as fatias de TC. O método é capaz de reconstruir as curvaturas da estruturas internas do paciente sem utilizar fatias próximas uma das outras. Os experimentos realizados mostram a eficácia do método proposto.

Palavras-chave. Interpolação de Imagem 3D, Transporte e Difusão de Informação, Fatia Virtual Inicial, Retoque em 3D.

\section{Introdução}

Tradicionalmente, o resultado da tomografia computadorizada (TC) mostra imagens de cortes transversais (fatias) da estrutura analisada. Essas fatias são impressas para que o médico possa visualizar os resultados do exame de TC, e elas estão distanciadas umas das outras de alguns milímetros, geralmente entre 1 e $10 \mathrm{~mm}$. A partir das imagens das fatias é possível também a visualização em 3D, que dispensa o profissional médico da tarefa mental de reconstruir as estruturas internas do corpo humano, auxiliando-o no processo de análise e diagnóstico.

Existe no mercado vários produtos visualizadores utilizando diferentes técnicas $[7,8,13,5]$, mas geralmente para apresentar uma imagem de alta qualidade, são

\footnotetext{
${ }^{1}$ sandrer@alfa.br

${ }^{2}$ C.A.Z. Barcelos é parcialmente suportada por recursos de pesquisa do CNPq

33celiazb@ufu.br, marcos@catalao.ufg.br, edna@ufu.br, gilberto@ufu.br, acpveiga@ufu.br
} 
utilizadas fatias próximas umas das outras, expondo o paciente a um maior tempo de incidência dos raios-X. A Figura 1 mostra a reconstrução de uma mandíbula humana usando a interpolação linear com diferentes quantidades de fatias para visualizar a mesma estrutura, sendo 26 fatias em (a) e 13 em (b).

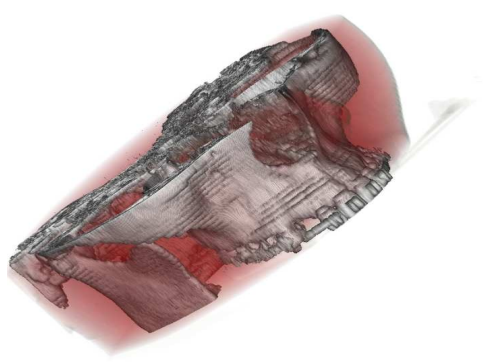

(a)

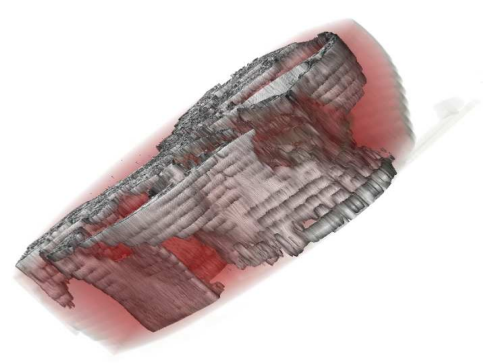

(b)

Figura 1: Visualização 3D utilizando o método da interpolação linear. (a) 26 fatias espaçadas de $2 \mathrm{~mm}$; (b) 13 fatias espaçadas de $4 \mathrm{~mm}$;

Motivado por um melhor balanceamento entre o tempo de exposição do paciente aos raios-X e a qualidade da visualização, este artigo apresenta o retoque digital como uma forma de interpolação em 3D das fatias de TC com imagens das estruturas do corpo humano. O retoque é feito via Equações Diferenciais Parciais (EDPs) não lineares visando produzir formas suaves e contornos bem definidos para a estrutura em questão sem a necessidade da utilização de uma grande quantidade de fatias.

Inicialmente, o uso de inpainting digital foi utilizado para a restauração de imagens fotográficas e filmes antigos, como o método proposto por Bertalmio et al. [4] que utiliza EDPs não lineares para restaurar imagens em 2D. Este trabalho utiliza esse mesmo processo de inpainting, mas para a realização de interpolação em 3D.

Este artigo está estruturado da seguinte maneira: a seção 2 apresenta alguns trabalhos correlatos sobre interpolação em 3D, a seção 3 mostra o método proposto, a seção 4 apresenta os experimentos realizados e os resultados obtidos. Finalmente, na seção 5 são apresentadas as conclusões.

\section{Trabalhos Correlatos}

Esta seção apresenta alguns trabalhos anteriores sobre interpolação de imagens em 3D, com os comentários sobre a contribuição deles no método proposto neste artigo.

Goshtasby et al. [9] propuseram um método de interpolação entre duas fatias, onde uma medida de similaridade define para um pixel em uma fatia, qual é o pixel correspondente na outra. Essa correspondência entre os pixels define o caminho para a interpolação linear. A mesma idéia é utilizada no método proposto, mas com um limiar de tolerância que é usado no processo de comparação. É possível que existam pixels sem correspondência nas fatias reais, criando regiões sem informações. 
Wang et al. [14] propuseram uma abordagem onde os espaços livres entre as fatias reais são divididos em pequenos cubos de pixels. A partir da análise das alterações do nível de cinza nos vértices do cubo, define-se a direção do processo de interpolação. Os resultados obtidos são similares aos da interpolação linear. Este trabalho utiliza essa idéia, pois as vizinhanças dos pixels são usadas para definir as conexões entre as fatias, ampliando o espaço de identificação de similaridades.

O método de Goshtasby et al. [9] e Wang et al. [14], além de outros como Bors et al. [6], consideraram a hipótese de que não existem mudanças abruptas nos contornos dos tecidos do corpo humano. Assim, eles consideraram a possibilidade da continuidade de um determinado tecido de uma fatia real na fatia adjacente. Essa é a hipótese fundamental do método proposto neste trabalho.

\section{Método Proposto}

Visando construir imagens em 3D com uma quantidade pequena de fatias de TC, aqui denominadas fatias reais, criam-se fatias virtuais entre duas fatias reais. $\mathrm{O}$ processo de criação é realizado em duas fases. Como as imagens das estruturas internas do corpo humano constantes nas fatias de TC apresentam grande semelhança entre si [9], conforme mostrado na Figura 2, a primeira fase consiste em criar uma representação inicial para as fatias virtuais, analisando essas similaridades.

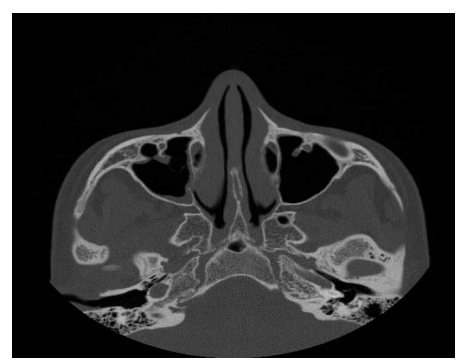

(a)

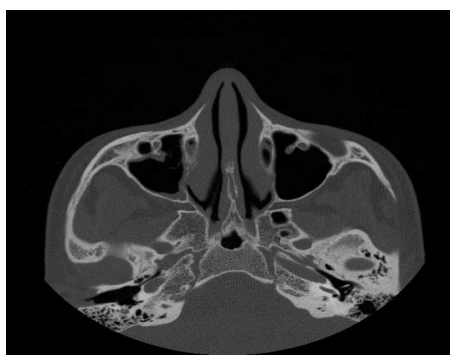

(b)

Figura 2: Duas fatias reais adjacentes.

Criada a representação inicial, o segundo passo é determinar as informações não constantes nessa representação inicial, usando o retoque digital em 3D.

\subsection{Representação inicial da imagem $3 \mathrm{D}$}

A representação inicial da imagem 3D é composta da justaposição de fatias reais e virtuais. Para criar as fatias virtuais entre duas fatias reais adjacentes, analisa-se a similaridade entre elas, que é obtida da seguinte forma: considere duas fatias reais adjacentes $F_{1}$ e $F_{2}$, e uma virtual $F_{v}$ entre elas. Um pixel $F_{v}(i, j)$ é dado por

$$
F_{v}(i, j)=\left\{\begin{array}{r}
F_{1}(i, j), \text { se } \operatorname{Min}\left(D_{F_{1}}, D_{F_{2}}\right)=D_{F_{1}} \text { e } D_{F_{1}}<T_{g} \\
F_{2}(i, j), \text { se } \operatorname{Min}\left(D_{F_{1}}, D_{F_{2}}\right)=D_{F_{2}} \text { e } D_{F_{2}}<T_{g} \\
A^{*}, \text { se } \operatorname{Min}\left(D_{F_{1}}, D_{F_{2}}\right)>T_{g}
\end{array}\right.
$$


onde $T_{g}$ é um limiar de tolerância,

$$
\begin{aligned}
D_{F_{1}}=\operatorname{Min}\left(\left|F_{1}(i, j)-F_{2}(i, j)\right|\right. & \left|F_{1}(i, j)-F_{2}(i+1, j)\right|, \\
\left|F_{1}(i, j)-F_{2}(i-1, j)\right| & \left|F_{1}(i, j)-F_{2}(i, j+1)\right|, \\
& \left.\left|F_{1}(i, j)-F_{2}(i, j-1)\right|\right), \\
D_{F_{2}}=\operatorname{Min}\left(\left|F_{2}(i, j)-F_{1}(i, j)\right|\right. & \left|F_{2}(i, j)-F_{1}(i+1, j)\right|, \\
\left|F_{2}(i, j)-F_{1}(i-1, j)\right| & \left|F_{2}(i, j)-F_{1}(i, j+1)\right|, \\
\mid & \left.\left|F_{2}(i, j)-F_{1}(i, j-1)\right|\right)
\end{aligned}
$$

e $A^{*}$ - valor atribuído para definir os pixels sem informação.

Para determinar $T_{g}$, é adotado a análise do desvio padrão dos níveis de cinza das fatias reais e um coeficiente $K, 0 \leq K \leq 1$, que define um nível de tolerância do processo. $T_{g}$ é dado por

$$
T_{g}=\frac{\sigma_{F_{n}}+\sigma_{F_{n+1}}}{2} K,
$$

onde $\sigma_{F_{n}}$ é o desvio padrão entre os níveis de cinza da fatia $F_{n}$.

A quantidade de fatias virtuais entre duas fatias reais adjacentes é dada em função da distância entre as fatias reais adjacentes e da resolução delas. No método proposto, todas as fatias virtuais possuem as mesmas informações. Aproveitando-se da hipóteses deste trabalho, outras variantes podem ser dadas para a geração das fatias virtuais. As regiões com os valores $A^{*}$ não contém informações e define o domínio de retoque, $D$, no próximo passo do processo.

O objetivo da representação inicial é diminuir o tamanho do domínio de retoque, diminuindo o tempo de processamento. Quanto mais similares forem duas fatias reais adjacentes, menor será $D$. Com o objetivo de aumentar a similaridade de duas fatias reais, propõe-se a aplicação de uma difusão anisotrópica, com conservação de bordas na imagem em 3D formada pelas fatias reais, antes da análise de similaridade.

O trabalho precursor de difusão anisotrópica foi proposto por Malik e Perona [11] para a remoção de ruídos. Após esse trabalho, vários outros surgiram na literatura para efetuar difusão com conservação de bordas $[1,2]$. Neste trabalho usa-se a equação de difusão proposta por Osher e Sethian [12] e descrita por

$$
I_{t}=|\nabla I| \operatorname{div}\left(\frac{\nabla I}{|\nabla I|}\right) .
$$

O balanceamento entre esses termos é feito de maneira seletiva, onde os pontos de borda e pontos de regiões uniformes dos objetos da imagem são tratados de maneira diferente. Barcelos et al. [3] também apresentou abordagem similar.

Embora o uso desse método de difusão seja a remoção de ruídos, pode-se utilizar o mesmo processo para aumentar o nível de similaridade entre duas fatias reais adjacentes. Este trabalho usa a equação de difusão proposta por Barcelos et al. [3] para esse novo fim, que é descrita por:

$$
I_{t}=g|\nabla I| \operatorname{div}\left(\frac{\nabla I}{|\nabla I|}\right)-(1-g)\left(I-I_{0}\right), \quad x \in R^{3} \text { e } I(x, 0)=I_{0},
$$


onde $g=g(|\nabla I|)$ e $I_{0}$ é a imagem original.

A função $g(s) \geq 0$ é uma função monotonicamente decrescente, satisfazendo às condições $g(0)=1$ e $g(s) \rightarrow 0$, quando $s \rightarrow \infty$. A função $g$ usualmente utilizada é

$$
g(\nabla I)=\frac{1}{\left(1+K_{g}|\nabla I|^{2}\right)},
$$

onde $K_{g}$ é uma constante.

A equação (3.5) faz um balanceamento, de forma seletiva, em pontos de borda e de regiões uniformes da imagem, que receberão a ação de difusão de forma diferenciada. A função $g$ acentua a ação nas regiões longe das bordas e atenua nas regiões das bordas. A Figura 3 ilustra os resultados obtidos pela aplicação do processo de difusão antes da análise de similaridade, que propicia a geração de uma representação inicial com regiões menores sem informação e mais bem definidas.

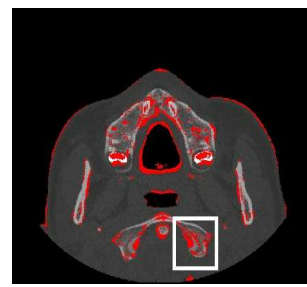

(a)

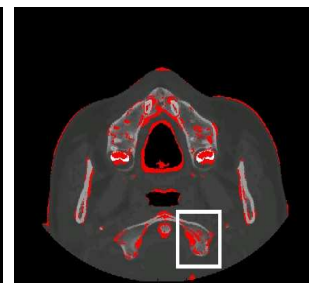

(b)

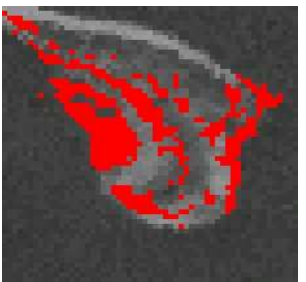

(c)

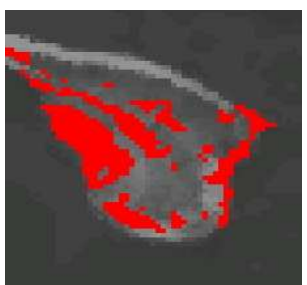

(d)

Figura 3: (a) e (b) Fatia virtual gerada sem e com o processo de suavização, respectivamente; (c) e (d) detalhes delas mostradas em (a) e (b), respectivamente.

\subsection{Interpolação utilizando retoque em $3 \mathrm{D}$}

O processo de retoque visa transportar as informações das regiões com informação da imagem 3D para dentro das regiões sem informação, D. Bertalmio et al. [4] propuseram um método de retoque divido em dois passos. O primeiro é o de transporte de informações da região externa para o interior de $D$, e o segundo é a difusão do conteúdo transportado. Barcelos et. al [2] propuseram uma modificação nesse processo de retoque, estendendo o passo de difusão para toda a imagem, contudo na porção da imagem fora de $D$, é utilizado o critério de conservação de bordas.

Este trabalho utiliza a abordagem proposta por Barcelos e Batista [2], onde o domínio de retoque são as regiões sem informação da imagem em 3D. O passo de transporte é feito por

$$
I_{t}=\nabla \mathcal{L}(I) \cdot \nabla^{\perp} I
$$

onde $I: \Omega \subset \mathbf{R}^{3} \longrightarrow[a, b] \subset \mathbf{R}$, e $D \subset \mathbf{R}^{3}$.

A difusão é realizada diferencialmente dentro e fora do conjunto $D$. Para os pontos externos ao conjunto $D$ a difusão é feita usando a equação (3.5), para os pontos internos a $D$, a difusão é realizada pela equação (3.4). 
A aplicação desse método de retoque permite que $D$ receba as informações transportadas de regiões mais distantes e não apenas da fronteira de $D$, resultando em contornos mais corretos.

\subsection{Detalhes da implementação}

Nesta seção são apresentados os detalhes de implementação para a interpolação em 3D. Cada elemento de $I_{i, j, k}$ é um valor inteiro correspondente ao nível de cinza da imagem $I\left(x_{i}, y_{j}, z_{k}\right)$. Denota-se $I\left(x_{i}, y_{j}, z_{k}, t_{n}\right)$ por $I_{i j k}^{n}$, onde $t_{n}=n \triangle t$. A derivada de $I$ em relação ao tempo $t$ (parâmetro de escala), $I_{t}$, calculado em $\left(x_{i}, y_{j}, z_{k}, t_{n}\right)$ é aproximada pelo método de Euler. A implementação numérica dos termos de transporte e difusão foram realizadas usando as técnicas de diferenças central [10]. Os termos de transporte e difusão são dados respectivamente por

$$
\begin{aligned}
L_{t}(I)= & \nabla \mathcal{L}(I) \cdot \nabla^{\perp} I \\
= & -\left(I_{x x y}+I_{y y y}+I_{z z y}\right) \cdot\left(I_{y}\right) \\
& +\left(I_{x x z}+I_{y y z}+I_{z z z}\right) \cdot\left(I_{x}+I_{z}\right)-\left(I_{x x x}+I_{y y x}+I_{z z x}\right) \cdot\left(I_{y}\right)
\end{aligned}
$$

e

$$
\begin{aligned}
& |\nabla I| \operatorname{div}\left(\frac{\nabla I}{|\nabla I u|}\right)= \\
& \frac{I_{x x}\left(I_{y}^{2}+I_{z}^{2}\right)+I_{y x}\left(I_{x}^{2}+I_{z}^{2}\right)+I_{z z}\left(I_{x}^{2}+I_{y}^{2}\right)}{I_{x}^{2}+I_{y}^{2}+I_{z}^{2}}-\frac{2 I_{x} I_{y} I_{x y}+2 I_{x} I_{z} I_{z x}+2 I_{y} I_{z} I_{y z}}{I_{x}^{2}+I_{y}^{2}+I_{z}^{2}} .(3 .
\end{aligned}
$$

Utilizando-se as condições de contorno de Neumann, calcula-se $I_{i j k}^{n+1}, n=1,2, \ldots, N$, pela discretização da equação (3.7) para o transporte e das equações (3.5) e (3.4) para difusão. Assim, obtém-se:

\section{Para transporte de informações}

$$
I_{i j k}^{n+1}=\left\{\begin{array}{r}
I_{i j k}^{n}+K_{t} \mathcal{L}_{t}\left(I_{i j k}^{n}\right), \forall x \in D \\
I_{i j k}^{n}, \forall x \in D^{C}
\end{array}\right.
$$

onde $\mathcal{L}_{t}(u)$ - obtido pela discretização da equação (3.9).

\section{Para difusão de informações}

$$
I_{i j k}^{n+1}=I_{i j k}^{n}+K_{d} \mathcal{L}_{d}\left(I_{i j k}^{n}\right), \quad \forall x \in D
$$

onde $\mathcal{L}_{d}(u)$ é obtido pela discretização de

$$
\mathcal{L}_{d}(v)=\left\{\begin{array}{r}
g|\nabla v| \operatorname{div}\left(\frac{\nabla v}{|\nabla v|}\right)-(1-g)\left(v-v_{I_{0}}\right), \forall v, v_{i_{0}} \in D^{C} \\
|\nabla v| \operatorname{div}\left(\frac{\nabla v}{|\nabla v|}\right), \forall v \in D
\end{array}\right.
$$

e $g$ é a função descrita na equação (3.6). 


\subsubsection{Algoritmos para a interpolação}

Os dois passos do método proposto neste trabalho podem ser implementados em um computador a partir de duas funções principais, cujos algoritmos são:

\section{Obtenção da Fatia Virtual Inicial}

1. Entrada dos parâmetros. São eles:

$K$ - limiar de tolerância; $\quad K_{g}$ - coeficiente de atenuação para a função $g$;

2. Aplicar o processo de difusão na imagem em 3D composta pelas fatias reais;

3. Obter duas fatias adjacentes $A$ e $B$, usadas para gerar a fatia virtual $C$;

4. Calcular o limite de tolerância $T_{g}$ utilizando-se a equação (3.3);

5. Para cada pixel $C_{i j}$ de $C$, faça:

Calcular $D_{F_{a}}$ utilizando-se a equação (3.2);

Calcular $D_{F_{b}}$ utilizando-se a equação (3.2);

Determinar o valor de $C_{i j}$ pela equação (3.1);

\section{Etapa de Transporte e Difusão}

1. Criar a imagem em 3D a partir da justaposição das fatias reais e virtuais;

2. Entrada dos parâmetros. São eles:

$M$ - total de iterações do processo; $\quad M_{t}$ - iterações de transporte de informações;

$M_{d}$ - número de iterações de difusão; $\quad K_{t}$ - Tamanho do passo de transp. e difusão;

$K_{d}$ - tamanho do passo na difusão; $\quad K_{g}$ - coeficiente de atenuação da função $g$;

3. Repita $M$ vezes

Repita $M_{t}$ vezes

Transporte de informação utilizando-se a equação (3.10);

Repita $M_{d}$ vezes

Difusão utilizando-se a equação (3.11);

\section{Resultados e Análises}

Para verificar a eficácia do método proposto, ele foi comparado com os métodos de Goshtasby et al. [9] e de interpolação linear. Este trabalho apresenta o resultado de um exame composto por 52 fatias. Essas fatias são imagens de 512 x 512 pixels com a resolução no tomógrafo configurada para que cada pixel represente $0,34 \mathrm{~mm}$ da estrutura mapeada na imagem. A quantidade de fatias virtuais entre cada par de fatias reais é obtida por

$$
N_{\text {Slices }}=\operatorname{Trunc}\left(\frac{d\left(F_{a}, F_{b}\right)}{0.34}\right)-1,
$$

onde $d\left(F_{a}, F_{b}\right)$ - distância entre as fatias $F_{a}$ e $F_{b}$, e

$\operatorname{Trunc}(x)$ - retorna o maior inteiro menor ou igual a $x$.

Foram criados subconjuntos com 50\%, 25\% e 12,5\% do tamanho original, mantendo sempre constante o afastamento entre as fatias em cada um dos subconjuntos. O objetivo dessa diferença de quantidade de informações entre os subconjuntos é medir a eficácia do método quando se diminui a quantidade de fatias para representar uma mesma estrutura. Foram utilizadas duas funções de transferências, uma para realçar a estrutura óssea e a outra para o rosto do paciente. O tempo de processamento, em segundos, para o subconjunto de $50 \%$ das fatias reais foi em: 9 


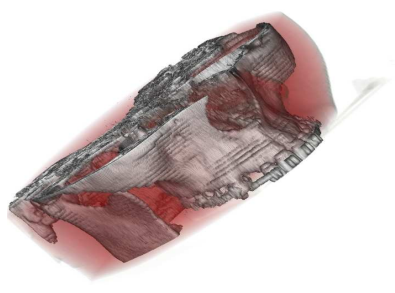

(a)

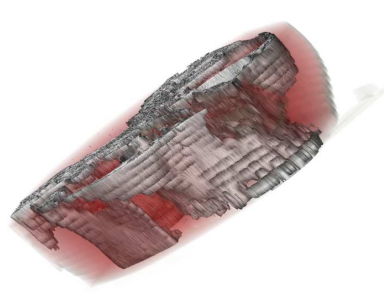

(d)

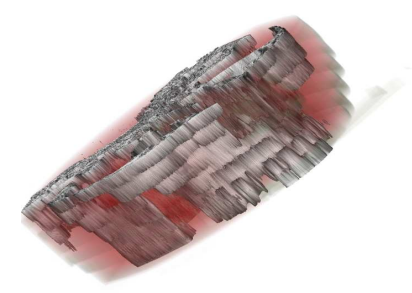

(g)

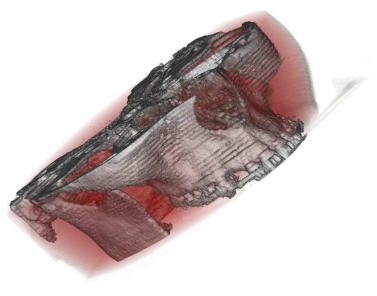

(b)

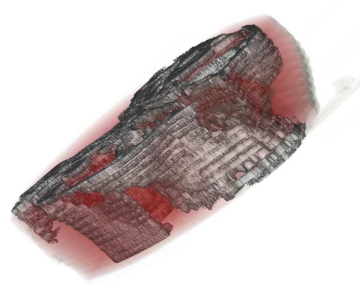

(e)

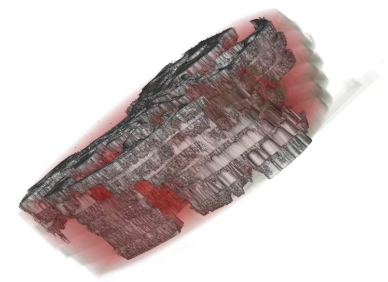

(h)

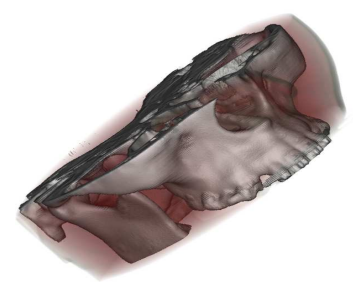

(c)

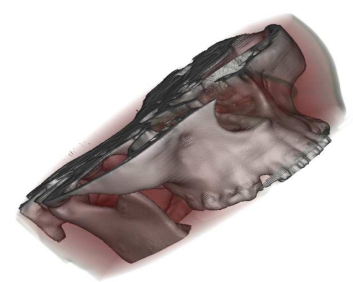

(f)

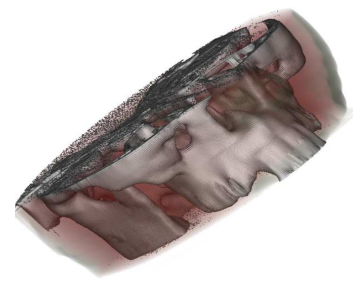

(i)

Figura 4: Resultados de aplicação dos métodos (colunas) para diferentes números de fatias (linhas).

para a interpolação linear, 16 para o método de Goshtasby et al. [9] e 184 para o método proposto.

As Figuras 4 e 5 mostram as imagens resultantes da interpolação linear na primeira coluna, Gohstasby et al. [9] na segunda coluna e do método proposto na terceira. No resultado original, as fatias estão afastadas em $1 \mathrm{~mm}$. Os parâmetros para as equações de transporte e difusão foram: $M=25, M_{t}=3, M_{d}=10$, $K_{t}=0.002, K_{d}=0.05$ e $K_{g}=0.6$.

As Figuras 4 (a) - (c) e 5 (a) - (c) mostram os resultados obtidos utilizando somente as fatias de números pares do conjunto original, isto é, o conjunto reduzido a $50 \%$, distância entre fatias reais de $2 \mathrm{~mm}$ e é necessário a geração de 5 fatias virtuais.

Pela análise visual pode-se observar que os três métodos produzem uma visualização satisfatória. Entretanto, o método proposto apresenta contornos mais suaves e precisos, tanto para as estruturas ósseas quanto para a visualização do resto. 


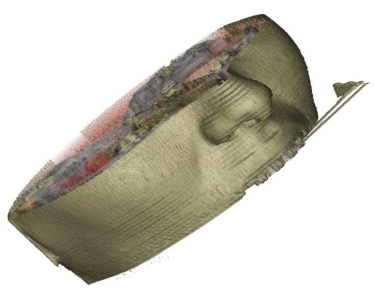

(a)

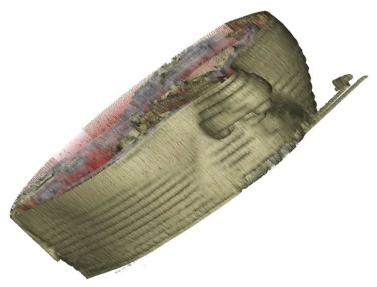

(d)

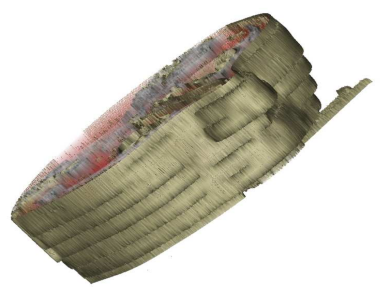

(g)

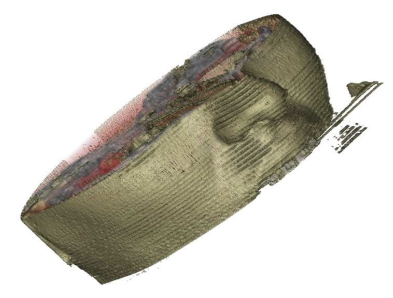

(b)

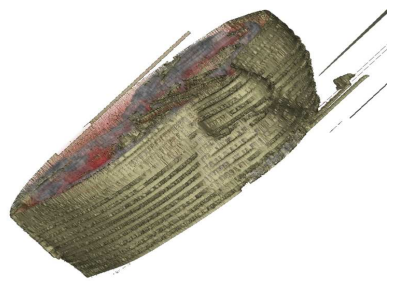

(e)

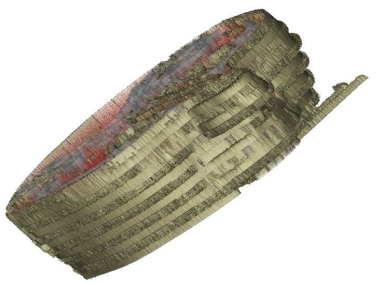

(h)

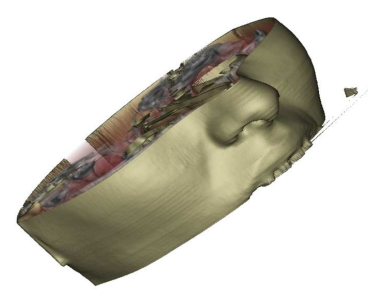

(c)

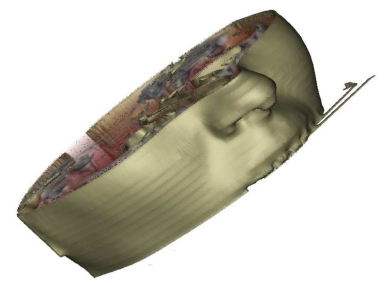

(f)

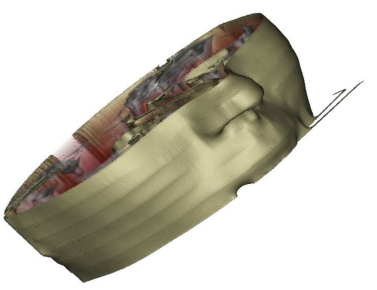

(i)

Figura 5: Resultados de aplicação dos métodos (colunas) para diferentes números de fatias (linhas).

As Figuras 4 (d) - (f) e 5 (d) - (f) mostram os resultados após a remoção de três fatias reais a cada conjunto de quatro, isto é, o conjunto de dados foi reduzido a $25 \%$ do tamanho original, distância entre fatias reais de $4 \mathrm{~mm}$ e é necessário a geração de 11 fatias virtuais. Com esse conjunto de dados, a visualização em 3D utilizando as treze fatias reais e as fatias virtuais interpoladas pelo método proposto ainda é satisfatória. O mesmo não se observa com os outros dois métodos.

As Figuras 4 (e) - (f) e 5 (e) - (f) mostram os resultados após a remoção de sete fatias reais a cada conjunto de oito, isto é, o conjunto de dados foi reduzido a $12.5 \%$ do tamanho original, com a distância entre fatias reais de $8 \mathrm{~mm}$ e é necessário a geração de 24 fatias virtuais.

Pode-se observar que o método proposto ainda permite o reconhecimento da estrutura, enquanto os outros métodos não permitem esse reconhecimento. Pode-se verificar também que quanto mais distantes estão as fatias reais umas das outras, mais nítida é a superioridade do método proposto. 


\title{
5. Conclusão
}

O uso de equações diferenciais não lineares para a interpolação em 3D produziu contornos suaves e bem definidos, possibilitando uma perfeita reconstrução de estruturas utilizando fatias reais intercaladas com fatias virtuais.

O processo de difusão possibilitou modelar satisfatoriamente a não existência de mudanças abruptas nos tecidos do corpo humano, validando a hipótese utilizada neste trabalho.

A aproximação torna possível uma reconstrução satisfatória mesmo utilizando apenas $5 \%$ de fatias reais e $95 \%$ de fatias interpoladas como é o caso mostrado nas Figuras 4 e 5 nas letras (g), (h) e (i), o que possibilita uma significativa redução do tempo de incidência de exposição dos pacientes aos raios-X, acarretando menor dano à saúde e menor custo para a realização dos exames.

\begin{abstract}
A visualization of the result of a computerized tomographic (CT) exam in $3 \mathrm{D}$ increases the quality of the medical diagnosis and thus the efficiency of the treatment. To obtain a high quality image it is necessary to obtain slices which are close to one another thus increasing the time span a patient is exposed to X-Ray. Motivated towards the goal of reaching an improved balance between patient's Xray exposition time and visualization quality, this research work presents digital inpainting as a form of 3D interpolation for CT slices used in the $3 \mathrm{D}$ visualization of the human body structures. The inpainting is carried out via non-linear partial differential equations. This work proposes a method capable of reconstructing the curvatures of the patient's internal structures without using slices that are close to one another. The experiments carried out show the proposed method's efficiency.
\end{abstract}

\section{Referências}

[1] L. Alvarez, P. Lions, J. Morel, Image selective smoothing and edge detection by nonlinear diffusion, SIAM Journal on Numerical Analysis, 29, No. 3 (1992), 845-866.

[2] C. Barcelos, M. Batista, Image restoration using digital inpainting and noise, Image and Vision Computing, (2007), 61-69.

[3] C. Barcelos, M. Boaventura, E. Silva Jr, A well-balanced flow equation for noise removal and edge detection, IEEE Transactions On Image Processing, 12, No. 7 (2003), 751-763.

[4] M. Bertalmío, et al., Image inpainting. in "Proceedings of SIGGRAPH", 2000, pp. 417-424. New Orleans, 2000.

[5] BLOX. BLOX. Website: http://sourceforge.net/projects/blox/, acessado em: 09/07/2007.

[6] G.A. Bors, L. Kechagias, L. Pitas, Binary morphological shape-based interpolation applied to 3 -d tooth reconstruction, IEEE Transactions on Medical Imaging, 21, No. 2 (2002), 100-108. 
[7] Corporate Headquarters Merge eFilm, AMIDE - Medical Imaging Data Examiner. 1126 S. 70th Street,Milwaukee, WI 53214-3151, USA. Website: amide.sourceforge.net, acessado em: 09/07/2007.

[8] General Electric Company, Website: www.ge.com, acessado em: 09/07/2007.

[9] A. Goshtasby, D. Turner, L. Ackerman, Matching of tomographic slices for interpolation, IEEE Transaction on Medical Imaging, 11, No. 4 (1992), 507516.

[10] R. Haralick, L. Shapiro, "Computer and Robot Vision", Addison-Wesley Publishing Company, Vol 1 e 2, 1993.

[11] J. Malik, P. Perona, Scale-space and edge detection using anisotropic diffusion, IEEE Transactions on Pattern Analisys and Machine Intelligence, 12, No. 7 (1988), 12-49.

[12] S. Osher, J. Sethian, Fronts propagating with curvature-dependent speed: algorithms based on Hamilton-Jacobi formulations, Journal of Computational Physics, 79 (1988), 12-49.

[13] SLICE. SLICE, Website: www.slicer.org, acessado em: 09/07/2007.

[14] Y. Wang, Z. Zhang, B. Guo, 3D image interpolation based on directional coherence, IEEE Workshop on Mathematical Methods in Biomedical Image Analysis, (2001), 195-202. 